of species, in particular for the BSC and for most geography-based hypotheses of speciation. Coyne and Orr could be accused of being 'admayrers', but that's not the worst thing one can be called, in my opinion. Unlike Mayr in his early work, the authors admit, albeit with palpable hesitation, that speciation can also occur without geographic isolation, in overlapping or 'sympatric' areas. Sympatric speciation, which for decades had all the caché of a four-letter word, is increasingly seen to be responsible for the origin of at least a minority of species.

Coyne and Orr also give renewed credence to models of speciation that emphasize a role for sexual or natural selection, rather than viewing the origin of species as little more than a by-product of isolation. One currently popular model, ecological speciation, de-emphasizes geographical settings and reaffirms the importance of local adaptations and natural selection in bringing about speciation. Reproductive isolation may be brought about by ecological processes, such as habitat fragmentation or the uneven distribution of resources. In such cases, interbreeding is prevented between populations that are limited - by behavioural, physiological and morphological adaptations - to a very particular set of prey items, for example, or a particular tree species that they need to build their nests. The absence of the homogenizing effect of gene flow between individuals, not through geography but by the divergence of adapted and ecologically important traits, possibly even in the same environment, then leads to reproductive isolation. In this way, natural selection can play a prominent role in speciation.

The most recent work, largely done on fish models, includes modern genomic approaches, such as the analysis of quantitative trait loci. It concludes that reproductive isolation and speciation may be a by-product of ecological differences and disruptive selection on a surprisingly small number of phenotypic traits, which are controlled by an equally small number of underlying genes. A role for selection can also be inferred from molecular genetic data on hybrid incompatibilities from models such as the fruitfly. These have yielded a few candidate 'speciation genes', which also show signs of natural selection having been at work.

Performing this demanding duet in masterly harmony, Coyne and Orr present an authoritative treatise on one of the most long-running debates in evolutionary biology. Speciation is an impressively up-to-date and enlightening synthesis - and an entertaining read. It deserves to join Darwin's On the Origin of Species, and Mayr's Systematics and the Origin of Species on the bookshelf of anyone who is interested in evolution.

Axel Meyer is in the Department of Biology,

University of Konstanz, 78457 Konstanz, Germany.

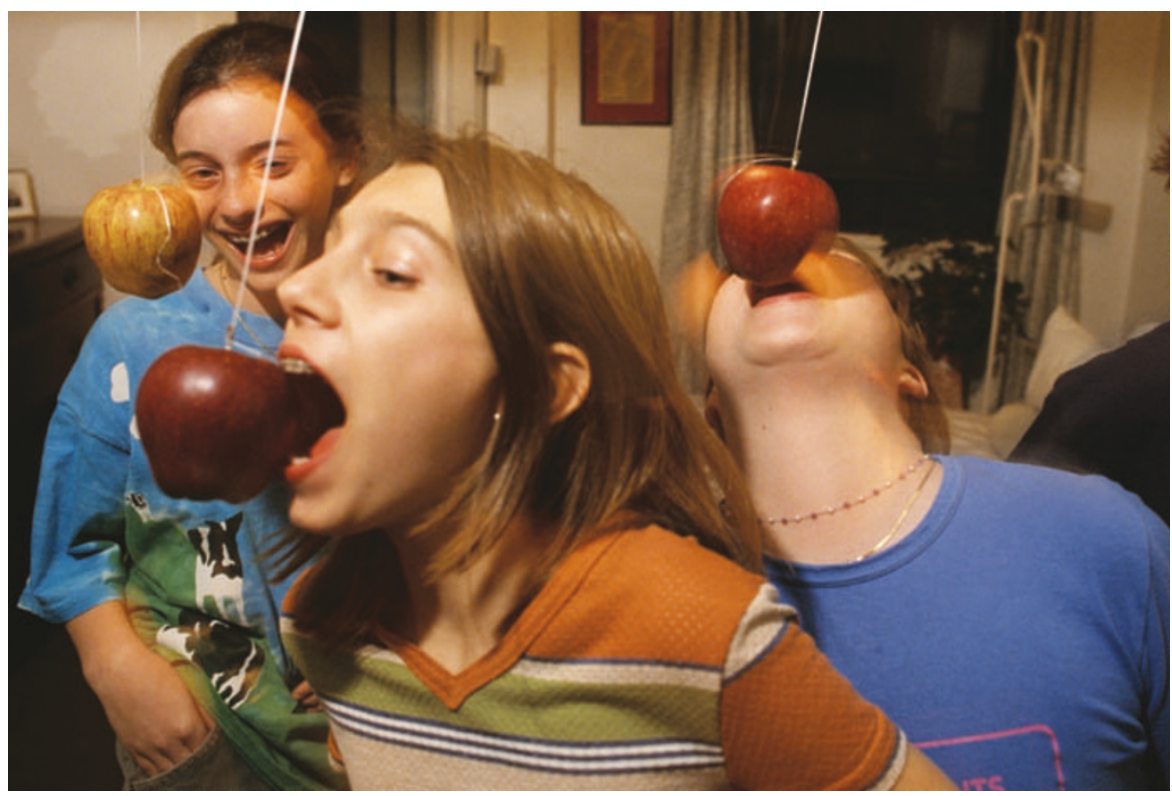

Grabbing a bite: the main role of teeth is to break down large items of food into smaller ones.

\section{Something to}

\section{chew on}

\section{Dental Functional Morphology: \\ How Teeth Work \\ by Peter W. Lucas \\ Cambridge University Press: 2004. 372 pp. \\ $\$ 130, \mathfrak{E} 75$}

\section{Daniel E. Lieberman}

Science has made substantial progress since Aristotle wrote (apparently without doing much research) that women have fewer teeth than men. The sheer volume of published research on teeth since may lead some to conclude that we have over-compensated for Aristotle's ignorance. Yet teeth merit all this attention because of their tremendous biological importance - not to mention the dreadful pain they can cause. Dental development and function are the focus of much clinical attention. And for evolutionary biologists, teeth are invaluable sources of information about taxonomy, phylogeny and many other aspects of animal biology.

There are already many excellent texts on dental function and development within the context of craniofacial development and clinical dentistry, as well as several good reviews of dental variation and evolution among vertebrates. But Dental Functional Morphology provides a fresh perspective on dental function. Peter Lucas's basic argument is that because the primary function of teeth is to reduce the size of food particles, dental morphology must be analysed in the context of how teeth fracture food, and how foods resist this. So the book reviews in detail many of the key mechanical properties of food, such as toughness and elasticity, which influence how teeth initially deform food items and generate cracks in them to break down large particles. Lucas then considers how variations in tooth size and shape influence this. He also includes more general reviews of dental and oral anatomy, and provides an excellent summary of the processes of chewing and oral transport, viewed from the perspective of the mechanical properties of food, such as particle size and stickiness.

This food's-eye view leads to numerous insights and interesting ideas, such as Lucas's theory of fracture scaling. Bigger animals have bigger teeth, whose surface areas might be predicted to scale with body mass to the power of 0.67 (because tooth area increases to the power of two, and body mass increases to the power of three, yielding a scaling ratio of $2 / 3$ ). Yet tooth surface area in mammals typically scales to the power of 0.61 . Why this is so remains elusive, but Lucas argues that fracture mechanics plays a role.

The argument is complex, but boils down to the observation that once a crack is initiated in an object, little additional energy is needed to finish the job, regardless of its size. Bigger foods fracture at relatively low stress, which has several implications. One is that bigger animals (assuming that they chew bigger food) need relatively less muscle force (as quantified by muscle cross-sectional area), so this should only increase to the power of 0.5 relative to body mass, although this has yet to be tested. If tooth surface area does not increase relative to bite force, then tooth surface area should also scale to 0.5 relative to body mass. But teeth scale to the power of 0.61 , so other factors must also influence tooth size, including other complex aspects of food mechanics also reviewed by Lucas. We can look forward to efforts to test this hypothesis and explore its implications.

Lucas does not consider in detail how dental function relates to tooth development and microstructure, or to the neuromuscular control of chewing. But readers interested in such topics as evolution, diet and ecology will enjoy his many other ideas about how vertebrate teeth work. The final chapter focuses mainly on mammals, creatively integrating 
biomechanics, anatomy, ecology and taxonomy in order to reconsider the evolution of dental adaptations for generating fractures in food for animals that eat insects, grasses, leaves, fruit and other animals. Primates, especially humans, get special attention. In general, we chew our food like other mammals, but the invention of cooking and other forms of food processing have drastically decreased the particle size and toughness of the food we eat. Palaeoanthropologists are still arguing about when cooking first evolved, but Lucas provides new reasons to suggest that the first species of the genus Homo, which had small teeth, was the first true chef of the animal world. Lucas calculates that your molars can be between $56 \%$ and $82 \%$ smaller to eat a cooked potato rather than a raw one, depending on whether you eat the skin and whether you roast or boil it.

Teeth often appear messy, confusing and dull to non-specialists, but Lucas succeeds in conveying his enthusiasm for the challenges of learning about the biology and ecology of organisms from such a small and humble organ. Although the book contains plenty of mechanics, the equations are presented clearly and well explained.

Lucas has filled the text with fascinating observations, humorous asides and wonderfully detailed footnotes. One particularly fun bonus is a flick-art animation running on the bottom corner of the book's pages that depicts the evolutionary transformation of a primitive single-cusped tooth into a human molar. Flicking through this cartoon will give your copy a thumb-worn look that Aristotle might have envied.

Daniel E. Lieberman is in the Department of Anthropology, Harvard University, 11 Divinity Avenue, Cambridge, Massachusetts 02138, USA.

\section{The geography of life}

\section{Foundations of Biogeography:}

Classic Papers with Commentaries edited by Mark V. Lomolino, Dov F. Sax \& James H. Brown

University of Chicago Press: 2004. 1,291 pp.

$\$ 135, £ 94.50$ ( $h b k) ; \$ 45, £ 31.50$ (pbk)

\section{Mark Williamson}

Collections of papers are useful both to undergraduates and their lecturers. Here is a set of 72 pieces on biogeography, 30 of which are excerpts from books. They were chosen and edited by a committee of 19 biologists, 13 of whom have contributed to the commentaries that precede the eight sections. Except for the first section, on early (mostly nineteenth century) classics, these are arranged by topic, which is helpful. In much the same way that a camel is a horse designed by a committee, the selection of topics has some strange bumps and depressions, but the resulting animal is nevertheless useful in the appropriate circumstances.

The editors suggest that biogeography is a recent discipline and that nine of the authors would not have called themselves biogeographers. But biogeography is a nineteenth-century term. Darwin wrote of the geographical distribution of organic beings, and Alfred Russel Wallace wrote of geographical zoology. And in the twentieth century, Robert MacArthur and Edward O. Wilson are in print saying "we both call ourselves biogeographers" and are "unable to see any real distinction between biogeography and ecology".

Biogeography might appear to be an interdisciplinary subject between biology and geography. It is certainly taught in both sets of departments, which once caused me a little difficulty as an external examiner because students had been taught essentially the same course twice. But biogeography is a branch of biology, of population and community ecology, and covers genetical and evolutionary topics as well as pure ecological ones. It is certainly an important branch, although the editors exaggerate its importance to Darwin and Wallace in discovering natural selection. It is nevertheless a branch with unusually fuzzy edges, differing from the related bits of biology largely by an emphasis on maps and geology.

With so many editors involved, the standards of the commentaries and the editing are both a bit variable, though it is mildly amusing to be told about "Louis Carroll's Through the Looking Glass" (two errors there) or that the muskrat "was introduced in 1905 into what was then Czechoslovakia". More seriously, in my view, the editorial board (who are all biologists) would have been well advised to seek the opinions of historians of science on the works from before 1950. Using facsimiles that have been standardized to a fixed page size means that some of the text is hard to read and some half-tones would have been better omitted. The cross-referencing is a bit weak, too. Some of the commentaries are excellent.

If your class reading list calls for something on Linnaeus, Buffon, de Candolle, von Humboldt and Hooker at one end of the time span, along with MacArthur and Wilson's theory and developments in the 1970s, this $2-\mathrm{kg}$ set will be very useful.

Mark Williamson is in the Department of Biology, University of York, York YO10 5DD, UK.

\section{Awash with art}

\section{Zeger Reyers saturates the senses with his installation Aqua Boogie.}

\section{Colin Martin}

The installation by Dutch artist Zeger Reyers in the basement of the GEM Museum of Contemporary Art in the Hague, the Netherlands, acknowledges the prior claim of water on the museum's site. The land was drained by previous generations.

Reyers' work frequently creates new biotopes, which display the vagaries of biological processes. At the 2003 Havana Biennial he released 200 white laboratory mice onto a 15-metre-long white tabletop covered with $5,000 \mathrm{~kg}$ of white porcelain. Their colouring made them difficult to spot, creating an installation that referenced protective adaptation in nature. Other previous installations covered interior spaces with living fungi.

To reach Aqua Boogie, visitors descend a staircase that has a view across a large pond outside, conveying a feeling of being below water level. They enter an irregular, 220-square-metre space, flooded with dark, non-reflecting water and criss-crossed with pine duckboards (right). As well as allowing viewers to explore the aqueous biotope more closely, this grid recalls Victory Boogie Woogie, a painting by Piet Mondrian, as the installation's title suggests.

The biotope affects many of the senses. "It has the thin fragrance of living water, like you smell when entering a canal," says Reyers. "The water also dampens sounds, making it a quiet, serene place." Beneath the surface, 35 leather carp, selected by Reyers because their dark colouring makes them practically invisible, go about unseen business. Like all Reyers' work, Aqua Boogie is accessible, but offers multiple levels of interpretation. It can disconcert viewers by challenging their perceptions of

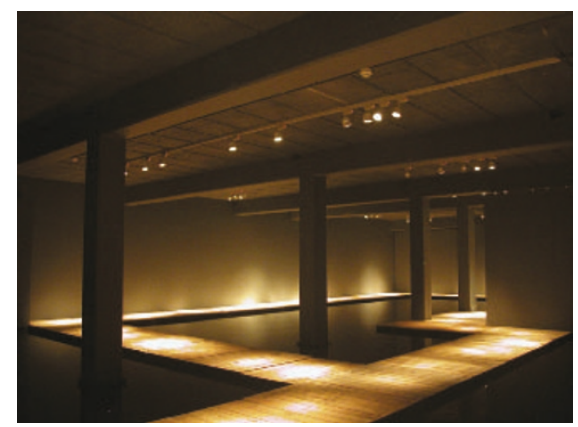

a familiar environment, experienced out of context.

On two occasions during the installation, which can be seen until 7 November, Reyers will recreate a 2001 work, Mussel Chair. A Parisian pavement café chair, encrusted with mussels after having been submerged in the Eastern Scheldt estuary for two years, will be brought to the museum, cleaned up and steamed. The cooked shellfish will be offered to visitors to eat, adding taste to the other sensory experiences offered by Aqua Boogie. Colin Martin is a writer based in London. 